\title{
On double integrals over spheres
}

\author{
R Burridge and $G$ Beylkin \\ Schlumberger-Doll Research, Old Quarry Road, Ridgefield, CT 06877-4108, USA
}

Received 7 April 1987, in final form 22 June 1987

\begin{abstract}
Formulae involving double integrals over spheres arise naturally in inverse scattering problems since the scattered data are measured in the space $R \times S^{2} \times S^{2}$. In this paper we derive a relation between differential forms on the space $\boldsymbol{S}^{n-1} \times \boldsymbol{S}^{n-1}$, and those on the space $I \times S^{n-2} \times S^{n-1}$, where $I$ is a real interval. Specifically,

$$
\mathrm{d} \xi \mathrm{d} \eta=\sin ^{n-2} \theta \mathrm{d} \theta \mathrm{d} \psi \mathrm{d} \nu
$$

$(\xi, \eta) \in \boldsymbol{S}^{n-1} \times \boldsymbol{S}^{n-1}$ and $(\theta, \psi, \nu) \in \boldsymbol{I} \times \boldsymbol{S}^{n-2} \times \boldsymbol{S}^{n-1}$. This allows us to derive the results of John relating the iterated spherical mean of a function to its spherical mean in a simple way; to obtain new inversion formulae for the Fourier and Radon transforms; to extend formulae for linearised inverse quantum scattering and diffraction tomography to the multifrequency case; and also to establish a relation between multifrequency diffraction tomography and seismic migration algorithms.
\end{abstract}

\section{Introduction}

Double integrals over spheres were investigated by John (1955) who derived a relation between the spherical mean of a function and the iterated spherical mean. Devaney $(1982 \mathrm{a}, \mathrm{b})$ derived certain inversion formulae in two- and three-dimensional spaces which are in the form of double integrals over spheres. This was then generalised by Beylkin $(1983 \mathrm{a}, \mathrm{b})$, who used John's results to obtain an inversion formula for the Fourier transform in terms of an integral over $S^{n-1} \times S^{n-1}$. Inversion formulae of this type are used in linearised inverse scattering problems and, in particular, provide a mathematical basis for diffraction tomography (Devaney and Beylkin 1984, Beylkin and Devaney 1985).

Double integrals over spheres arise naturally in typical inverse scattering problems where one point on the unit sphere represents the unit normal of an incident plane wave and a second point on the sphere represents the scattering direction at which a measurement is made. Ideally the experiment is repeated for all possible directions of incidence and scattering, and inversion formulae naturally involve integration over these spheres of directions. When frequency varies in such an experiment it is natural to integrate over this variable as well (or the dual time variable) giving formulae involving integration over $\boldsymbol{R} \times \boldsymbol{S}^{2} \times \boldsymbol{S}^{2}$.

In the lemma of $\S 2$ we derive a differential relation between the volume elements on $\boldsymbol{S}^{n-1} \times \boldsymbol{S}^{n-1}$ and a space which can be described as $[0, \pi] \times \boldsymbol{S}^{n-1} \times \boldsymbol{S}^{n-2}$. Using this relation and a few simple variants we derive a number of inversion formulae and describe their application. First, we give a simple derivation of the results of John (1955). We then proceed to obtain in $\S 3$ certain inversion formulae for the Fourier transform and the 
Radon transform. In $\S 4$ using results of $\S 3$ we derive some formulae in inverse scattering theory extending results of Devaney (1982a). We then discuss diffraction tomography where the inversion formula of $\S 3$ yields a generalisation to what can be called multifrequency diffraction tomography. The same inversion formula is also used in the derivation of migration algorithms used in inverting seismic prospecting data (Beylkin and Burridge $1987 \mathrm{a}, \mathrm{b}$ ), thus establishing a relationship between multifrequency diffraction tomography and seismic migration.

\section{Relation between differential forms on spheres}

Let $\xi, \eta, \nu$ be three coplanar unit vectors in $\boldsymbol{R}^{n}$ and suppose that

$$
\lambda \xi+\mu \eta=r \nu
$$

where $\lambda \geqslant \mu>0$ are fixed positive constants and $r=|\lambda \xi+\mu \eta|$ is a scalar function of $\lambda, \mu, \xi$ and $\eta$ :

$$
r^{2}=\lambda^{2}+2 \lambda \mu \cos \theta+\mu^{2}
$$

Let

$$
\cos \alpha=\xi \cdot \nu \quad \cos \beta=\eta \cdot \nu \quad \cos \theta=\xi \cdot \eta
$$

so that

$$
\alpha+\beta=\theta .
$$

Then it is clear that

$$
\alpha=\alpha(\theta) \quad \beta=\beta(\theta) .
$$

Also, from (2.2) we have

$$
r \mathrm{~d} r=-\lambda \mu \sin \theta \mathrm{d} \theta
$$

We shall prove the following.

Lemma. Consider differential forms $\mathrm{d} \xi, \mathrm{d} \eta, \mathrm{d} \nu$ on unit $(n-1)$-dimensional spheres over which the corresponding vectors vary. Then

$$
\mathrm{d} \xi \mathrm{d} \eta=\sin ^{n-2} \theta \mathrm{d} \theta \mathrm{d} \psi \mathrm{d} \nu
$$

where $\psi$ varies over an $(n-2)$-dimensional sphere and $\theta \in[0, \pi]$. The unit vector $\psi$ lies in the plane of $\xi, \eta, \nu$ and is perpendicular to $\nu$ :

$$
\begin{aligned}
& \xi=\cos \alpha \nu-\sin \alpha \psi \\
& \eta=\cos \beta \nu+\sin \beta \psi
\end{aligned}
$$

Once we have (2.7) other related formulae follow easily. Thus from (2.7) and (2.6) follows

$$
\mathrm{d} \xi \mathrm{d} \eta=-\frac{1}{\lambda \mu} \sin ^{n-3} \theta r \mathrm{~d} r \mathrm{~d} \psi \mathrm{d} \nu
$$

where $\sin \theta$ is expressible in terms of $r$ (see (2.2)) and $r \in[\lambda-\mu, \lambda+\mu]$. It is natural to 
normalise $r$ by $\lambda+\mu$. Thus we may define $\rho$ by

$$
r=(\lambda+\mu) \rho \quad \mathrm{d} r=(\lambda+\mu) \mathrm{d} \rho
$$

to obtain the equivalent relation

$$
\mathrm{d} \xi \mathrm{d} \eta=-\frac{(\lambda+\mu)^{2}}{\lambda \mu} \sin ^{n-3} \theta \rho \mathrm{d} \rho \mathrm{d} \psi \mathrm{d} \nu \quad \gamma \leqslant \rho \leqslant 1,
$$

where $\gamma=(\lambda-\mu) /(\lambda+\mu)$.

Proof of lemma. Let $\psi$ be a unit vector perpendicular to $\nu$ and lying in the plane of $\xi, \eta$. Choose coordinates so that $\nu$ lies along the $x_{n}$ axis, and $\xi, \eta, \nu, \psi$ lie in the $\left(x_{n-1}, x_{n}\right)$ plane. Then from (2.8) it is clear that

$$
\left.\begin{array}{l}
\mathrm{d} \xi_{k}=\cos \alpha \mathrm{d} \nu_{k}-\sin \alpha \mathrm{d} \psi_{k} \\
\mathrm{~d} \eta_{k}=\cos \beta \mathrm{d} \nu_{k}+\sin \beta \mathrm{d} \psi_{k}
\end{array}\right\} \quad \text { for } k=1, \ldots, n-2
$$

and so

$$
\mathrm{d} \xi_{k} \mathrm{~d} \eta_{k}=\sin \theta \mathrm{d} \nu_{k} \mathrm{~d} \psi_{k} \quad \text { for } k=1, \ldots, n-2 .
$$

Here we have used (2.4), (2.12) and the addition formula for sine.

In the $\left(x_{n-1}, x_{n}\right)$ plane let us denote by $\mathrm{d} \xi^{\prime}, \mathrm{d} \nu^{\prime}, \mathrm{d} \eta^{\prime}, \mathrm{d} \psi^{\prime}$ the infinitesimal angular displacements of those vectors. Then

$$
\begin{aligned}
& \mathrm{d} \xi^{\prime}=\mathrm{d} \nu^{\prime}-\mathrm{d} \alpha \\
& \mathrm{d} \eta^{\prime}=\mathrm{d} \nu^{\prime}+\mathrm{d} \beta
\end{aligned}
$$

and so

$$
\mathrm{d} \xi^{\prime} \mathrm{d} \eta^{\prime}=\mathrm{d} \nu^{\prime}(\mathrm{d} \alpha+\mathrm{d} \beta)=\mathrm{d} \nu^{\prime} \mathrm{d} \theta
$$

Thus, on combining (2.13) and (2.15) we have

$$
\mathrm{d} \xi \mathrm{d} \eta=\mathrm{d} \nu \mathrm{d} \psi \sin ^{n-2} \theta \mathrm{d} \theta
$$

where

$$
\begin{aligned}
& \mathrm{d} \xi=\mathrm{d} \xi_{1} \ldots \mathrm{d} \xi_{n-2} \mathrm{~d} \xi^{\prime} \\
& \mathrm{d} \eta=\mathrm{d} \eta_{1} \ldots \mathrm{d} \eta_{n-2} \mathrm{~d} \eta^{\prime} \\
& \mathrm{d} \nu=\mathrm{d} \nu_{1} \ldots \mathrm{d} \nu_{n-2} \mathrm{~d} \nu^{\prime} \\
& \mathrm{d} \psi=\mathrm{d} \psi_{1} \ldots \mathrm{d} \psi_{n-2} .
\end{aligned}
$$

\section{Applications}

\section{Iterated spherical means}

Following John (1955, p 78) let us consider the iterated spherical mean of a function $f$ :

$$
M(x, \lambda, \mu)=\frac{1}{\Omega_{n}^{2}} \int_{\boldsymbol{S}^{n-1} \times \boldsymbol{S}^{n-1}} f(x+\lambda \xi+\mu \eta) \mathrm{d} \xi \mathrm{d} \eta
$$


where $\Omega_{n}=2 \pi^{n / 2} / \Gamma\left(\frac{1}{2} n\right)$ is the surface area of the $(n-1)$ sphere. By (2.9) this is

$$
M(x, \lambda, \mu)=\frac{1}{\lambda \mu \Omega_{n}^{2}} \int_{\lambda-\mu}^{\lambda+\mu} \int_{s^{n-1}} \int_{s^{n-2}} f(x+r \nu) \sin ^{n-3} \theta \mathrm{d} \psi \mathrm{d} \nu r \mathrm{~d} r .
$$

Hence, integrating $\psi$ over $S^{n-2}$, we have

$$
M(x, \lambda, \mu)=\frac{\Omega_{n-1}}{\lambda \mu \Omega_{n}^{2}} \int_{\lambda-\mu}^{\lambda+\mu} \int_{S^{n-1}} f(x+r \nu) \mathrm{d} \nu \sin ^{n-3} \theta r \mathrm{~d} r .
$$

Now the area of the triangle with sides $\lambda, \mu, r$ and angle $\theta$ opposite $r$ is

$$
\frac{1}{4} H(r) \equiv \frac{1}{2} \lambda \mu \sin \theta=\frac{1}{4}[(\lambda+\mu+r)(\lambda+\mu-\lambda)(\mu+r-\lambda)(r+\lambda-\mu)]^{1 / 2}
$$

and so

$$
\begin{aligned}
M(x, \lambda, \mu) & =\frac{2 \Omega_{n-1}}{(2 \lambda \mu)^{n-2} \Omega_{n}^{2}} \int_{\lambda-\mu}^{\lambda+\mu} r \mathrm{~d} r \int_{s^{n-1}} \mathrm{~d} \nu H^{n-3}(r) f(x+r \nu) \\
& =\frac{2 \Omega_{n-1}}{(2 \lambda \mu)^{n-2} \Omega_{n}} \int_{\lambda-\mu}^{\lambda+\mu} H^{n-3}(r) I(x, r) r \mathrm{~d} r
\end{aligned}
$$

where

$$
I(x, r)=\frac{1}{\Omega_{n}} \int_{S^{n-1}} f(x+r \nu) \mathrm{d} \nu
$$

is the ordinary spherical mean. Equation (3.5) is formula (4.9c) of John (1955).

\section{Integral over all of space}

For future applications it is useful to express an integral over $\boldsymbol{R}^{n}$ in terms of an integral over $\boldsymbol{R} \times \boldsymbol{S}^{n-1} \times \boldsymbol{S}^{n-1}$. Thus consider

$$
I=\int_{R^{n}} f(p) \mathrm{d} p
$$

Then

$$
\begin{aligned}
I=\int_{0}^{\infty}|p|^{n-1} \mathrm{~d}|p| \int_{S^{n-1}} f(|p| \nu) \mathrm{d} \nu & \\
& =\frac{1}{C \Omega_{n-1}} \int_{0}^{\infty}|p|^{n-1} \mathrm{~d}|p| \int_{S^{n-1}} f(|p| \nu) \mathrm{d} \nu \frac{1}{1-\gamma} \int_{\gamma}^{1} W(\rho) \mathrm{d} \rho \int_{S^{n-2}} \mathrm{~d} \psi
\end{aligned}
$$

where

$$
C=\frac{1}{1-\gamma} \int_{\gamma}^{1} W(\rho) \mathrm{d} \rho .
$$

Now using (2.11) we have

$$
\mathrm{d} \rho \mathrm{d} \psi \mathrm{d} \nu=-\frac{\lambda \mu}{(\lambda+\mu)^{2}} \frac{\mathrm{d} \xi \mathrm{d} \eta}{\rho \sin ^{n-3} \theta}
$$


where $\xi$ and $\eta$ are defined as in the lemma. Using (3.10) in (3.8) and setting $r=\rho(\lambda+\mu)$ we have

$$
\begin{aligned}
I=\frac{\lambda \mu}{\Omega_{n-1}(\lambda+\mu)} & \frac{1}{C(1-\gamma)} \int_{0}^{\infty}|p|^{n-1} \mathrm{~d}|p| \\
& \times \int_{S^{n-1} \times S^{n-1}} f\left(|p| \frac{(\lambda \xi+\mu \eta)}{r}\right) \frac{W(r /(\lambda+\mu))}{r \sin ^{n-3} \theta} \mathrm{d} \xi \mathrm{d} \eta
\end{aligned}
$$

where

$$
r=|\lambda \xi+\mu \eta|
$$

Next set

$$
|p|=r k \quad \mathrm{~d}|p|=r \mathrm{~d} k
$$

to get

$$
\begin{aligned}
I=\frac{\lambda \mu}{2 \Omega_{n-1}(\lambda+\mu)} & \frac{1}{C(1-\gamma)} \int_{-\infty}^{\infty}|k|^{n-1} \mathrm{~d} k \\
& \times \int_{\boldsymbol{S}^{n-1} \times \boldsymbol{S}^{n-1}} f(k(\lambda \xi+\mu \eta)) \frac{r^{n-1} W(r /(\lambda+\mu))}{\sin ^{n-3} \theta} \mathrm{d} \xi \mathrm{d} \eta
\end{aligned}
$$

where the integral over $k$ has been extended to the whole real axis and

$$
\sin ^{n-3} \theta=\left[1-(\xi \cdot \eta)^{2}\right]^{(n-3) / 2}
$$

Inverse Fourier transform on the space $\boldsymbol{R} \times \boldsymbol{S}^{n-1} \times \boldsymbol{S}^{n-1}$

We can now formulate the following.

Theorem 1. Let $g$ be a function on $\boldsymbol{R}^{n}$ and $\hat{g}$ its Fourier transform. Then

$$
\begin{gathered}
g(y)=\frac{1}{4(2 \pi)^{n} \Omega_{n-1}} \int_{-\infty}^{\infty}|k|^{n-1} \mathrm{~d} k \int_{\boldsymbol{s}^{n-1} \times \boldsymbol{s}^{n-1}} \mathrm{~d} \xi \mathrm{d} \eta \frac{|\xi+\eta|^{n-1}}{\left[1-(\xi \cdot \eta)^{2}\right]^{(n-3) / 2}} \\
\times W\left(\frac{1}{2}|\xi+\eta|, y\right) \hat{g}(k \xi+k \eta) \exp [\mathrm{i}(k \xi+k \eta) \cdot y]
\end{gathered}
$$

where $W$ is an arbitrary function such that

$$
\int_{0}^{1} W(\rho, y) \mathrm{d} \rho=1
$$

and where $\Omega_{n}=2 \pi^{n / 2} / \Gamma\left(\frac{1}{2} n\right)$ is the surface area of the unit sphere in $\boldsymbol{R}^{n}$.

Theorem 1 can be generalised further and leads to

Theorem 2. Let $g$ be a function on $\boldsymbol{R}^{n}$ and $\hat{g}$ its Fourier transform. Let $\lambda=\lambda(y)$ and $\mu=$ $\mu(y)$ be two positive functions on $\boldsymbol{R}^{n}$. Then

$$
\begin{aligned}
g(y)=\frac{1}{4(2 \pi)^{n} \Omega_{n-1}} & \int_{-\infty}^{\infty}|k|^{n-1} \mathrm{~d} k \\
& \times \int_{\boldsymbol{s}^{n-1} \times \boldsymbol{S}^{n-1}} \mathrm{~d} \xi \mathrm{d} \eta b(y, \xi, \eta) \hat{g}(k \lambda \xi+k \mu \eta) \exp [\mathrm{i}(k \lambda \xi+k \mu \eta) \cdot y]
\end{aligned}
$$


where

$b(y, \xi, \eta)=\frac{|\lambda \xi+\mu \eta|^{n-1}}{\left[1-(\xi \cdot \eta)^{2}\right]^{(n-3) / 2}} \frac{2 \lambda \mu}{(\lambda+\mu)} \frac{1}{1-\gamma(y)} W\left(\frac{|\lambda \xi+\mu \eta|}{\lambda+\mu}, y\right)$

and

$$
\gamma(y)=\frac{|\lambda(y)-\mu(y)|}{\lambda(y)+\mu(y)} .
$$

Here, for each $y, W(\rho, y)$ is an arbitrary function of $\rho$ such that

$$
\frac{1}{1-\gamma(y)} \int_{\gamma(y)}^{1} W(\rho, y) \mathrm{d} \rho=1 .
$$

Theorem 1 follows from theorem 2 by setting $\lambda(y)=\mu(y)=1$.

Proof of theorem 2. The inversion formula for the Fourier transform is an integral over $\boldsymbol{R}^{n}$ :

$$
g(y)=\frac{1}{(2 \pi)^{n}} \int_{R^{n}} \hat{g}(p) \exp (\mathrm{i} p \cdot y) \mathrm{d} p
$$

Now use (3.14) with

$$
f(p)=\frac{1}{(2 \pi)^{n}} \hat{g}(p) \exp (\mathrm{i} p \cdot y)
$$

and set $C=1$. Notice that since $y$ is only a parameter in (3.23) we may take $f, W, \lambda, \mu$ and $\gamma$ to depend upon $y$ in (3.7)-(3.14). Such dependence is explicitly indicated in (3.16)-(3.21).

Remark 1. Formulae (3.16) and (3.18) can be modified to have integration with respect to $k$ over the positive part of the $k$ axis; for example, instead of $(3.18)$ we obtain

$$
\begin{aligned}
g(y)=\frac{1}{2(2 \pi)^{n} \Omega_{n-1}} & \int_{0}^{\infty} k^{n-1} \mathrm{~d} k \int_{\boldsymbol{S}^{n-1} \times \boldsymbol{S}^{n-1}} \mathrm{~d} \xi \mathrm{d} \eta \\
& \times b(y, \xi, \eta) \hat{g}(k \lambda \xi+k \mu \eta) \exp [\mathrm{i}(k \lambda \xi+k \mu \eta) \cdot y] .
\end{aligned}
$$

Remark 2. To clarify the role of the function $W$, let us restrict integration over $k$ to an interval $\left[k_{\min }, k_{\max }\right]$ and compute

$$
\begin{aligned}
g_{\mathrm{B}}(y)=\frac{1}{2(2 \pi)^{n} \Omega_{n-1}} \int_{k_{\min }}^{k_{\max }} k^{n-1} \mathrm{~d} k \int_{\boldsymbol{s}^{n-1} \times \boldsymbol{S}^{n-1}} \mathrm{~d} \xi \mathrm{d} \eta \\
\quad \times b(y, \xi, \eta) \hat{g}(k \lambda \xi+k \mu \eta) \exp [\mathrm{i}(k \lambda \xi+k \mu \eta) \cdot y] .
\end{aligned}
$$


Using (2.11), (3.15), (3.19) and integrating over $\psi$, we have from (3.25)

$$
\begin{aligned}
g_{\mathrm{B}}(y)=\frac{1}{(2 \pi)^{n}} \int_{k_{\min }}^{k_{\max }} k^{n-1} \mathrm{~d} k \frac{1}{1-\gamma(y)} \int_{\gamma(y)}^{1} \mathrm{~d} \rho \int_{\boldsymbol{S}^{n-1}} \mathrm{~d} \nu \\
\quad \times W(\rho, y) \rho^{n}(\lambda+\mu)^{n} \hat{g}(k \rho(\lambda+\mu) \nu) \exp [\mathrm{i} k \rho(\lambda+\mu) \nu \cdot y] .
\end{aligned}
$$

Changing the order of integration in (3.26) we arrive at

$$
g_{\mathrm{B}}(y)=\frac{1}{1-\gamma(y)} \int_{y(y)}^{1} \mathrm{~d} \rho W(\rho, y) g_{\rho}(y)
$$

where

$$
g_{\rho}(y)=\frac{1}{(2 \pi)^{n}} \int_{p(\lambda+\mu) k_{\min } \leqslant|p| \leqslant \rho(\lambda+\mu) k_{\max }} \mathrm{d} p \hat{g}(p) \exp (\mathrm{i} p \cdot y) .
$$

Formula (3.27) can be interpreted as a superposition of band-limited reconstructions $g_{\rho}$. Thus, the choice of $W$ determines the spatial frequency content of the final result $g_{\mathrm{B}}$. If $k_{\min }=0$ and $k_{\max }=\infty$ it is easy to see that $g_{\mathrm{B}}(y)=g(y)$.

Relation to the Radon transform. Let us point out the relation of (3.16) and (3.18) to the Radon transform. We define the Radon transform on the space $\boldsymbol{R} \times \boldsymbol{S}^{n-1} \times \boldsymbol{S}^{n-1}$ as follows

$$
v(t, \xi, \eta)=(R g)(t, \xi, \eta)=\int_{R^{n}} g(x) \delta[t-(\lambda \xi+\mu \eta) \cdot x] \mathrm{d} x .
$$

When defined in this way $v$ contains redundant information. Relations (3.16) and (3.18) allow us to invert this transform directly. We have

$$
(\hat{R} g)(k, \xi, \eta)=\int_{-\infty}^{+\infty}(R g)(t, \xi, \eta) \exp (\mathrm{i} k t) \mathrm{d} t=\hat{g}(k \lambda \xi+k \mu \eta)
$$

Let us define the backprojection operator $R^{*}$ as

$$
\left(R^{*} v\right)(y)=\left.\frac{1}{\Omega_{n}^{2}} \int_{\mathbf{S}^{n-1} \times \mathbf{S}^{n-1}} \mathrm{~d} \xi d \eta b(y, \xi, \eta) v(t, \xi, \eta)\right|_{t=(\lambda \xi+\mu \eta) \cdot y}
$$

where $b$ is described in (3.19). Also let us define the one-dimensional convolutional operator $K$ as given by

$$
(K h)(t)=\int_{-\infty}^{\infty} K\left(t-t^{\prime}\right) h\left(t^{\prime}\right) \mathrm{d} t^{\prime}
$$

where

$$
K(t)=\frac{\Omega_{n}^{2}}{4 \Omega_{n-1}(2 \pi)^{n}} \int_{-\infty}^{\infty}|k|^{n-1} \exp (\mathrm{i} k t) \mathrm{d} k
$$

The operator $K$ in spaces of odd dimension is just the operator of differentiation of order $n-1$. In spaces of even dimension in addition to differentiation it contains the Hilbert transform. 

obtain

Given the definitions of the operators $R^{*}$ and $K$ we substitute $(3.30)$ into $(3.18)$ to

$$
g(y)=\left(R^{*} K v\right)(y)
$$

the inversion formula for the Radon transform defined in (3.29).

\section{Inverse scattering in the Born approximation}

The inverse Fourier transform on the space $\boldsymbol{R} \times \boldsymbol{S}^{n-1} \times \boldsymbol{S}^{n-1}$ as described in theorem 1 has a straightforward application to the inverse scattering problem in the Born approximation. In fact, this was one of the problems that motivated the study of such integrals in the first place. Indeed, the scattering data are measured in the space $R \times S^{2} \times S^{2}$, where $R$ is the frequency domain (or time domain, depending on a particular application) and unit spheres represent incident and scattered directions.

Consider the three-dimensional Lippmann-Schwinger integral equation describing quantum-mechanical scattering of a plane wave by a potential $V$,

$$
\Psi(k, \xi, x)=\exp (\mathrm{i} k \xi \cdot x)-\frac{1}{4 \pi} \int \mathrm{d} y \frac{\exp (\mathrm{i} k|x-y|)}{|x-y|} V(y) \Psi(k, \xi, y)
$$

where the potential $V$ is such that for large $|x|$ the solution of (4.1) has the asymptotic behaviour

$$
\Psi(k, \xi, x)=\exp (\mathrm{i} k \xi \cdot x)+\frac{\exp (\mathrm{i} k|x|)}{|x|} A(k, \xi, \eta)+o\left(\frac{1}{|x|}\right)
$$

where $\eta=x /|x|$ and $A(k, \xi, \eta)$ is the scattering amplitude. Given $\Psi(k, \xi, x)$ satisfying (4.1), $A(k, \xi, \eta)$ can be written as

$$
A(k, \xi, \eta)=-\frac{1}{4 \pi} \int \mathrm{d} x \exp (-\mathrm{i} k \eta \cdot x) V(x) \Psi(k, \xi, x) .
$$

Usually one can measure only $|A(k, \xi, \eta)|^{2}$, the differential cross section, but occasionally $A$ itself can be measured in actual experiments. The inverse problem consists in finding the potential, given the scattering amplitude. It is also of interest to see what can be recovered if only the differential cross section is measured.

To linearise this inverse problem we use the Born approximation which amounts to replacing the wavefunction $\Psi(k, \xi, x)$ by the incident wavefunction $\exp (i k \xi \cdot x)$. We then obtain from (4.3) a linear relation between the potential and the scattering amplitude,

$A(k, \xi, \eta)=-\frac{1}{4 \pi} \int \mathrm{d} x \exp (-\mathrm{i} k \eta \cdot x) V(x) \exp (\mathrm{i} k \xi \cdot x)=-\frac{1}{4 \pi} \hat{V}(k \eta-k \xi)$.

Similarly, within the Born approximation,

$$
|A(k, \xi, \eta)|^{2}=\frac{1}{(4 \pi)^{2}} \hat{Q}(k \eta-k \xi)
$$

where $\hat{Q}$ is the Fourier transform of the so-called interatomic distance function,

$$
Q(x)=\int V(x+y) \bar{V}(y)
$$


The validity of the Born approximation in the context of the quantum-mechanical scattering can be justified under the assumption of smallness of the potential or for high energies (frequencies) for a given potential.

Our inversion formula provides a direct reconstruction of the potential and the interatomic distance function for the linearised inverse problem. Indeed, applying theorem 1 specialised to dimension $n=3$ (see also $\S 3$, remark 1) with $g \equiv V$ and using (4.4) we have

$$
\begin{aligned}
V(y)=-\frac{1}{(2 \pi)^{3}} & \int_{0}^{\infty} k^{2} \mathrm{~d} k \int_{s^{2} \times s^{2}} \mathrm{~d} \xi \mathrm{d} \eta|\xi-\eta|^{2} \\
& \times W\left(\frac{1}{2}|\xi-\eta|, y\right) A(k, \xi, \eta) \exp [\mathrm{i}(k \eta-k \xi) \cdot y]
\end{aligned}
$$

and again with $g \equiv Q$ and using (4.5) we have

$$
\begin{aligned}
Q(y)=\frac{1}{2 \pi^{2}} \int_{0}^{\infty} & k^{2} \mathrm{~d} k \int_{\boldsymbol{S}^{2} \times \boldsymbol{s}^{2}} \mathrm{~d} \xi \mathrm{d} \eta|\xi-\eta|^{2} \\
& \times W\left(\frac{1}{2}|\xi-\eta|, y\right)|A(k, \xi, \eta)|^{2} \exp [\mathrm{i}(k \eta-k \xi) \cdot y]
\end{aligned}
$$

where $W$ is an arbitrary function such that

$$
\int_{0}^{1} W(\rho, y) \mathrm{d} \rho=1 .
$$

These formulae are important generalisations of inversion formulae described by Devaney (1982a), and their importance stems from the fact that reconstruction is performed using all the available frequencies while the results in Devaney (1982a) are single-frequency reconstructions. The formulae derived here are also related to the tracetype formulae, but these results will be described elsewhere.

The arbitrary function $W$ can be used to emphasise different parts of the spectrum of spatial frequencies in the reconstruction provided the scattering amplitude is measured over an interval of energies (see $\S 3$, remark 2 ) and, also, can be used in practice to cut off energies with poor signal-to-noise ratios. The simplest choice, however, is to set $W=1$.

A scattering experiment usually produces data for a single incident direction and a sequence of such experiments usually accounts for all the data collected. The order of integration in (4.7) and (4.8) can be changed to accommodate this. Thus the most natural way of accomplishing it would be to make the last (outer) integration that over the incident direction, so that for each of the experiments there is a partial reconstruction and the last step is to average such partial reconstructions.

\section{Multifrequency diffraction tomography and migration algorithms}

More delicate use of the results decribed in theorems 1 and 2 can be made in the linearised inverse scattering problem in acoustics and elasticity. In fact, the generalisation given by theorem 2 specifically addresses the elastic case where $\lambda \neq \mu$ because $\mathrm{P}$ and $\mathrm{S}$ waves travel at different speeds (see Beylkin and Burridge 1987a, b). Also complications arise for two other reasons. First, it is preferable to solve the inverse problem in acoustics and elasticity for point sources rather than plane waves since that is how most of the experiments are carried out. Second, it is preferable to solve the inverse problem as a problem of finding a perturbation from a known spatially varying medium. This is because in acoustics and elasticity the single scattering approximation is valid only for small perturbations. 
Both of these complications (as compared with the quantum-mechanical inverse scattering problem) have been resolved. The problem with point sources was reduced more or less routinely to the problem with incident plane waves. The solution in a variable background medium can be obtained in a systematic way if we restrict ourselves to reconstructing discontinuities of the perturbations. In this case the theory of pseudodifferential operators allows us to reduce the equations to a point where theorem 1 or 2 can be used (see Beylkin 1985). The derivation of migration algorithms using theorems 1 and 2 for the reconstruction of several parameters in acoustics and elasticity will appear separately (Beylkin and Burridge 1987a, b).

In the context of ultrasound diffraction tomography, as described in Devaney and Beylkin (1984) and Beylkin and Devaney (1985), the plane-wave scattering amplitude is expressed as an integral over point sources and receivers which are located on arbitrary surfaces surrounding the region of interest. Within the linearised theory the plane-wave scattering amplitude is related to the unknown perturbation via an expression similar to (4.4). Therefore, it is just a matter of using the inversion formulae of theorems 1 and 2 instead of inversion formulae for fixed frequency to obtain reconstruction formulae of what can be called multifrequency diffraction tomography. It is then clear that multifrequency diffraction tomography and migration in exploration seismology are essentially the same algorithms if understood through theorems 1 and 2 .

\section{References}

Beylkin G 1983a The fundamental identity for iterated spherical means and the inversion formula for diffraction tomography and inverse scattering J. Math. Phys. 24 1399-400

_ 1983b Iterated spherical means in linearised inverse problems Conf. on Inverse Scattering: Theory and Applications ed. J B Bednar et al (Philadelphia: SIAM)

- 1985 Imaging of discontinuities in the inverse scattering problem by inversion of a causal generalised Radon transform J. Math. Phys. 26 99-108

Beylkin G and Burridge R 1987a Multiparameter inversion for acoustic and elastic media Expanded Abstract, 57 th Ann. Int. Meeting of the Society of Exploration Geophysicists, New Orleans

$1987 \mathrm{~b}$ Linearised inverse scattering problem of acoustics and elasticity, in progress

Beylkin G and Devaney A J 1985 Mathematical framework of diffraction tomography Research Note (Ridgefield, CT: Schlumberger-Doll Research)

Devaney A J 1982a Inversion formula for inverse scattering within the Born approximation Opt. Lett. 1111-2

- 1982b A filtered backpropagation algorithm for diffraction tomography Ultrasonic Imaging 4 336-50

Devaney A J and Beylkin G 1984 Diffraction tomography using arbitrary transmitter and receiver surfaces Ultrasonic Imaging 6 181-93

John F 1955 Plane Waves and Spherical Means (New York: Interscience) 